\title{
Quantitative evaluation of thin uranium foils using $x$-ray radiography
}

Muhammad Abir, Daniel Wachs, Dennis Keiser Jr., Fahima Islam

October 2017

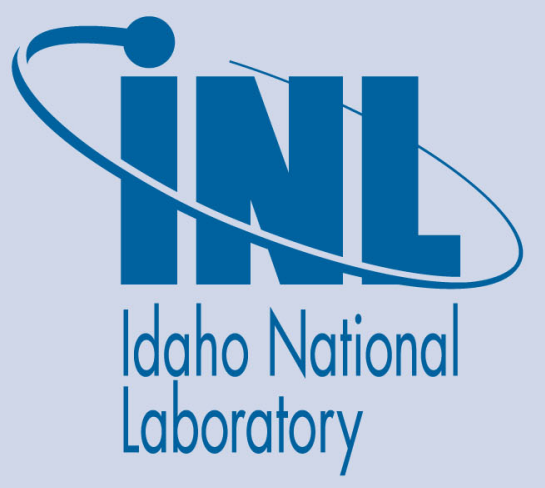

The INL is a U.S. Department of Energy National Laboratory operated by Battelle Energy Alliance 


\title{
Quantitative evaluation of thin uranium foils using $x$ - ray radiography
}

\author{
Muhammad Abir, Daniel Wachs, Dennis Keiser Jr., Fahima Islam
}

October 2017

Idaho National Laboratory Idaho Falls, Idaho 83415

http://www.inl.gov

Prepared for the

U.S. Department of Energy

Under DOE Idaho Operations Office

Contract DE-AC07-05ID14517 


\title{
Quantitative evaluation of thin uranium foils using X-ray radiography
}

\author{
Muhammad Abir ${ }^{\mathrm{a}, \mathrm{c}, *}$, Fahima Islam ${ }^{\mathrm{b}}$, Daniel Wachs ${ }^{\mathrm{c}}$, Dennis Keiser $\mathrm{Jr}^{\mathrm{c}}$ \\ a Massachusetts Institute of Technology, 77 Massachusetts Ave., Building-NW13-261, Cambridge, MA 02139-4307, USA \\ ${ }^{\mathrm{b}}$ Missouri University of Science and Technology, Department of Nuclear Engineering, 301 W. 14th St., Rolla, MO 65409, USA \\ c Idaho National Laboratory, P.O. Box-1625, MS 6188, Idaho Falls, ID 83415, USA
}

The quality of research reactor fuel plates fabricated for irradiation testing purposes is dependent on the dis-tribution of uranium bearing fuel phase within the fuel meat. This distribution is routinely monitored in plate type fuel to avoid excessive variability in local power that can lead to 'hot spots' that increase the probability for fuel failure. X-ray imaging has been routinely used to successfully eliminate this failure mode. However, characterization of this variability at a much higher resolution is also critical to support fuel performance as-sessment and traditional analysis techniques are not sufficient to meet research needs. An accurate and re-producible method for using X-ray imaging to non-destructively locate the distributed fuel phase and to evaluate the local uranium density in a fuel plate has been developed and is discussed in this paper. Four mini research reactor fuel plats were radiographed along with a well characterized U-8Mo step wedge using identical radio-graphy exposure conditions. The intensity distribution inside the foil were then correlated with the intensity values of the U-8Mo step wedge. Considering the attenuation and density, the spatial distribution of effective fuel thickness was estimated and eventually the distribution of average fuel loading for each fuel plate was estimated. Results demonstrate that the X-ray imaging can be utilized to estimate fuel loading variation in the fuel plate samples and can serve as a representative method for the assessment of nuclear fuels plates for research pur-poses.

\section{Introduction}

The US Department of Energy's National Nuclear Security Agency's (DOE-NNSA) Materials Management and Minimization (M3) program is developing fuels to replace highly enriched fuel with low enriched fuel (Abir et al., 2016). A variety of irradiation experiments have been conducted over the last two decades to study the in-pile behavior of both uranium molybdenum alloy (U-Mo) and uranium silicide compounds $\left(\mathrm{U}_{3} \mathrm{Si}_{2}\right)$ in dispersion type fuel forms for this purpose (Abir et al., 2016). In dispersion fuel systems, the fuel particles are dispersed in an aluminum matrix (as shown in Fig. 1) that forms the fuel meat. The fuel meat is encapsulated in aluminum alloy cladding that protects the fuel meat from interaction with the reactor coolant. The performance of a given fuel system is measured by its resistance to dimensional change and its ability to retain fission products throughout its operational lifetime.

Irradiation testing is conducted to support this performance assessment by enabling measurement of the nuclear fuel plate's thickness increase as a function of fissile consumption (described as either burnup or fission density). This growth is caused by the growth of the fuel phase located within the fuel meat. The extent of growth is a function of the specific material's ability to manage both solid and gaseous fission products produced when the fissile material fissions. The extent of growth is readily determined by measuring the fuel plate thickness before and after irradiation but the specific growth of the fuel phase depends on the amount of fuel phase located within the plate at the given point of measurement. This local 'fuel loading' must therefore be accurately estimated to support swelling estimates of the desired accuracy.

The fuel phase is typically distributed inhomogeneously within the fuel meat. The degree of inhomogeneity is a strong function of the manufacturing process used to produce the plates, which is immature for most new fuel designs. The distribution in the fuel meat is susceptible to a variety of imperfections, including agglomeration of fuel particles, irregular fuel meat shapes, incomplete or excessive rolling reduction, etc.

While the determination of maximum fuel loading is crucial to avoid excessive local power (e.g. 'hot spots') during irradiation, a finer description of the fuel loading is required to inform post irradiation evaluation of fuel swelling. Therefore, prior to inserting test fuel plates 


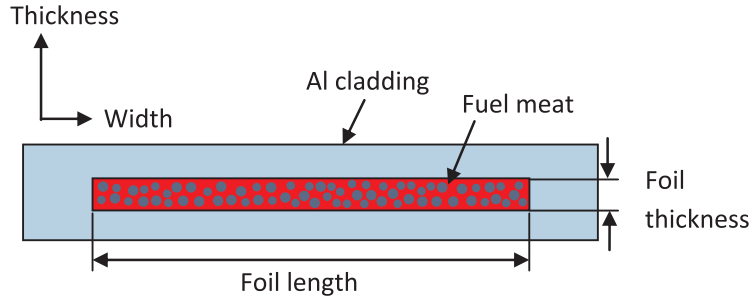

Fig. 1. Schematic of cross section of dispersion fuel (Meyer et al., 2014).

into the reactor, a reliable non-destructive inspection is necessary to inform the post-test experiment analysis. The fuel loading in a fuel plate is typically described by the effective overall and local fuel thickness as determined by quantitative analysis of radiographic X-ray film. A quantitative analysis process developed for this purpose is outlined in detail in this paper. The method is demonstrated on a set of fuel plates whose irradiation performance is of particular interest and cannot be adequately described without this baseline characterization.

$\mathrm{X}$-ray imaging is a useful non-destructive technique for examining internal structures of materials, determining atomic compositions and densities. Typically, X-ray imaging is performed on each fuel plate at the end of production (although it can also be used to guide processing during interim process steps). It provides a two-dimensional projection image after it interacts with the sample. The fluctuation of intensities in the X-ray image corresponds to the attenuation variation through the object. For images with homogeneous samples consisting of a single material, variation in pixel brightness can only be caused by variations in the sample thickness. For images with multiple overlapping materials, however, variation in pixel brightness depends on the relative thicknesses and attenuation properties of each material and the individual attenuation of each material is unknown because there can be many combinations of thicknesses and attenuation coefficients that produce the same gray level in an image.

While X-ray photons travel through a medium, a portion of them will interact with atomic electrons and attenuates before arriving at the detector. The attenuation of X-rays differs depending on the characteristics of the medium being imaged. X-ray radiography inherently contains the attenuation information for imaged objects because the brightness of a pixel in a radiograph is proportional to the intensity of the radiation absorbed by that pixel $(I)$. The magnitude of the radiation absorbed by the detector pixel depends on the intensity of the radiation being generated and the attenuation of the radiation as it travels through the medium on its path to the detector. By considering only uncollided radiation, the Beer-Lambert Law can be used to determine this quantity based on initial intensity $\left(I_{0}\right)$ of X-rays, linear attenuation coefficients $(\mu)$ of the object, and path lengths $(x)$ of X-rays:

$I=I_{0} e^{-\mu(\rho, Z, E) x}$

where the linear attenuation coefficient $(\mu)$ is a function of the density $(\rho)$, and the atomic number of matter $(Z)$, and the effective energy of the $\mathrm{X}$-ray photons $(E)$. X-rays are polychromatic in nature and the attenuation depends on the energy of the incident photons. For an inhomogeneous object with different attenuating medium (1) then becomes:

$I(E)=\int_{0}^{E} I_{0}(E)\left[-e^{\sum_{j=1}^{J} \mu_{j}(\rho, Z, E) x_{j}}\right] d E$

where $j$ indicates different material, each of their own attenuation coefficient and thickness. Due to the polychromatic nature of X-rays, analytical determination of fuel phase thickness is a non-trivial problem. Therefore, the thickness variation of the fuel is obtained by normalizing against a U-8Mo step wedge of known thicknesses and known density imaged next to the sample.
In this work, an accurate image analysis technique is employed to calculate fuel phase thickness hence to estimate the fuel loading variations in the fuel meat. The images of the foils are acquired using X-ray imaging technique and several processing are performed prior to calculating the fuel loading variations accurately. The recipe for the image analysis steps is described in Section 3.

\section{Experiment description}

\subsection{Fuel characteristics}

The Reduced Enrichment for Research and Test Reactors Advanced Fuel Development Project (RERTR-AFD) designs several dispersion fuel miniplates to be irradiated in the Advanced Test Reactor (ATR). The miniplates are fabricated at the Idaho National Laboratory (INL) and other RERTR international partners. Four dispersion fuel plates selected from the RERTR- 8 and RERTR-9B experiments are used in this work to assess the fuel loading variations in the fabricated fuel. These include two $\mathrm{U}_{3} \mathrm{Si}_{2}$ miniplates (U0R040 and U0R060) from the RERTR-8 experiment and two U-7Mo miniplates (R6R018 and R6R038) from the RERTR-9B experiment.

The silicide fuel plates in the RERTR-8 experiment consist of a $\mathrm{U}_{3} \mathrm{Si}_{2}$ fuel phase dispersed in an aluminum matrix. The $\mathrm{U}_{3} \mathrm{Si}_{2}$ fuel powder is produced by first arc melting uranium and silicon pucks. The pucks are then ground and sieved to produce the desired powder size distribution. The fuel meat is encapsulated in Al-6061 alloy cladding using the historic manufacturing process originally developed to fabricate fuel for the INL's Advanced Test Reactor (ATR) and ORNL's High Flux Isotope Reactor (HFIR). The fuel plates were fabricated at INL using lab scale processes modeled after the standard commercial processes. Historical fuel performance testing has demonstrated acceptable performance in dispersed $\mathrm{U}_{3} \mathrm{Si}_{2}$ fuel plates if the volume loading fraction stays below $45 \%$ (a maximum uranium loading of $4.8 \mathrm{gU} / \mathrm{cm}^{3}$ ).

The U-7Mo fuels in RERTR-9B dispersion mini-plates were fabricated using a variant of the standard process. The atomized fuel powder was fabricated at INL using a rotating electrode atomizer. The alloy powder was then blended with the aluminum based matrix material (Al-2 Si alloy and or Al-3.5 Si alloy) and then compacted into a pellet. The pellet is inserted into an Al-6061 frame and seal welded in an inert glovebox. The assembly is then hot rolled to final thickness. Additional hot pressing may be used to improve the bonding at clad/clad and fuel/ clad interfaces as desired. The acceptable limit of fuel loading fraction for the RERTR-9B fuels are yet to be determined. The properties of the fuel plates are shown in Table 1.

\subsection{Image acquisition}

The fuel plates are placed on X-ray films including all necessary lead markers to identify each plate ID. Since low energy X-rays cannot penetrate through the plates due to excessive attenuation in uranium, high energy X-rays are used to penetrate X-rays through the fuel plate. Depleted uranium molybdenum (DU-8Mo) calibration step wedge (photograph shown in Fig. 2) with varying thicknesses are imaged next to the fuel as a density standard as shown in Fig. 3. An aluminum shim of thickness equal to twice the nominal cladding thickness in the fuel

Table 1

Properties of inspected fuels.

\begin{tabular}{lll}
\hline Fuel properties & R6R018/R6R038 & U0R040/U0R060 \\
\hline Fuel composition & U-7Mo/Al-3.5Si & $\mathrm{U}_{3} \mathrm{Si}_{2}$ \\
Bulk density in fuel phase $\left(\mathrm{g} / \mathrm{cm}^{3}\right)$ & 17.1 & 12.2 \\
$\begin{array}{l}\text { Nominal Uranium loading in fuel meat } \\
\left(\mathrm{gU} / \mathrm{cm}^{3}\right)\end{array}$ & 8.0 & 4.8 \\
Enrichment (\%) & 58.14 & 74.86 \\
Nominal fuel meat thickness $(\mathrm{cm})$ & 0.0508 & 0.0635 \\
\hline
\end{tabular}




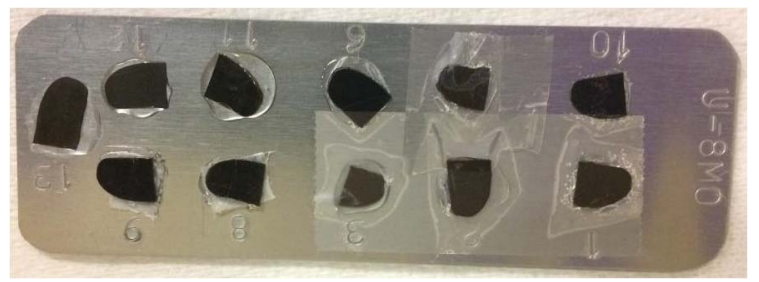

Fig. 2. Shows the depleted uranium molybdenum (U-8Mo) step wedge standard.

zone area of the plate is included with the density standard. The DU8Mo step wedge is used as a standard since the linear attenuation coefficients of $\mathrm{U}-8 \mathrm{Mo}, \mathrm{U}-7 \mathrm{Mo}$ and $\mathrm{U}_{3} \mathrm{Si}_{2}$ are very similar and do not vary significantly as a function of energy.

The X-ray films are digitized using Genesis NEO s60 film digitizer. The digitizer uses a cold cathode lamp as light source that can measure optical density up to 4.7 with spatial resolution up to $600 \mathrm{dpi}$. The digitized radiographs are shown in Fig. 3.

\section{Image processing and analysis}

\subsection{Intensity inhomogeneity correction}

Similar features in the image should have global intensity uniformity irrespective to the location in the image. Balancing uniform lighting across the entire X-ray image is a difficult problem due to the conical nature of the X-ray beam. Because of the inherent imperfections of the image formation process, spurious intensity variation or shading is usually present throughout the image as shown in Fig.4(a). Prospective shading correction can be obtained by capturing a bright image (or flat field image) of an empty field and a dark image with shutter closed and performs processing using (3) commonly referred as flat field correction (Tomaževič et al., 2002):

Corrected Imge $=\frac{\text { Raw Image-Dark Image }}{\text { Bright Image-Dark Image }} * K$

where $K$ is a normalization constant for restoration of desired gray level range. However, the perspective shading correction requires the specimen to be removed from the field of view which may not be possible in many situations. Furthermore, if the prospective correction cannot handle object dependent shading, the correction can be performed using a retrospective algorithm (Tomaževič et al., 2002). Retrospective correction can be performed by constructing an estimated background using different techniques if the background is not recorded (Oberholzer et al., 1996; Reyes-Aldasoro, 2009; Beckers et al., 1993; Likar, 2000). Shading appears as a slowly varying (low frequency) signal (Oberholzer et al., 1996) in the image which can be estimated using low pass or rank filers such as Gaussian, median filter, etc. The shading component is then subtracted or divided from the original image to obtain the corrected image. The original image is divided by the shading component for linear image acquisition, whereas the shading components should be subtracted for the logarithmic image acquisition (Russ, 2004). The median filter removes the features leaving just the background variation as shown in Fig. 4(b).

\subsection{Beam hardening correction}

Since polychromatic X-rays were used to acquire the images, low energy X-ray photons are attenuated more than high-energy photons as the X-rays pass through the sample. This phenomenon is commonly known as beam hardening which causes an underestimation of the attenuation values for thicker specimens. This causes a non-linear response in X-ray absorption with material thickness. Therefore, correction is needed to produce linear response and reduce inaccuracies arising from beam hardening. The U-8Mo step wedge density standard made of depleted uranium and $8 \mathrm{wt} \%$ molybdenum (U-8wt $\% \mathrm{Mo}$ ) is used to estimate the beam hardening correction and estimate the thickness and fuel loading in the fuel plate. The composition of the density standard is assumed to be a reasonable approximation for any $\mathrm{U}-\mathrm{Mo}$ and $\mathrm{U}_{3} \mathrm{Si}_{2}$ fuels. The geometry of density standard is shown in Fig. 5 with a minimum thickness of $0.0202 \mathrm{~cm}$ and a maximum thickness of $0.0584 \mathrm{~cm}$. With the absence of beam hardening, the relationship should be linear. The graph in Fig. 6 shows the deviation of the pixel brightness from linearity, indicating the degree of beam hardening with increasing wedge thickness. The effect of beam hardening is reduced by using a polynomial correction function that eliminates the non-linear relationship.

The corrected absorption values are calculated based on a voltage specific correction equation:

$\ln \left(\frac{I_{0}}{I_{\text {corr }}}\right)=\ln \left(\frac{I_{0}}{I_{\text {measured }}}\right)+\varepsilon_{v}\left[\ln \left(\frac{I_{0}}{I_{\text {measured }}}\right)\right]$

where the correction equation $\left(\varepsilon_{v}\right)$ is derived using third order polynomial least square fit:

$\varepsilon_{v}=A_{0}+A_{1}\left[\ln \left(\frac{I_{0}}{I_{\text {measured }}}\right)\right]+A_{2}\left[\ln \left(\frac{I_{0}}{I_{\text {measured }}}\right)\right]^{2}+A_{3}\left[\ln \left(\frac{I_{0}}{I_{\text {measured }}}\right)\right]^{3}$

where $A_{0}, A_{1}, A_{2}$, and $A_{3}$ are the polynomial correction values. The beam hardening correction using the polynomial correction function provides a linear relationship between wedge thickness and absorption values as shown in Fig. 6. It is necessary to mention that, although eleven standards (Fig. 2) were radiographed, only six density standards ranging from $0.0202 \mathrm{~cm}$ up to $0.0584 \mathrm{~cm}$ are used for calibration purpose. This is because the thicknesses greater than $0.0584 \mathrm{~cm}$ are
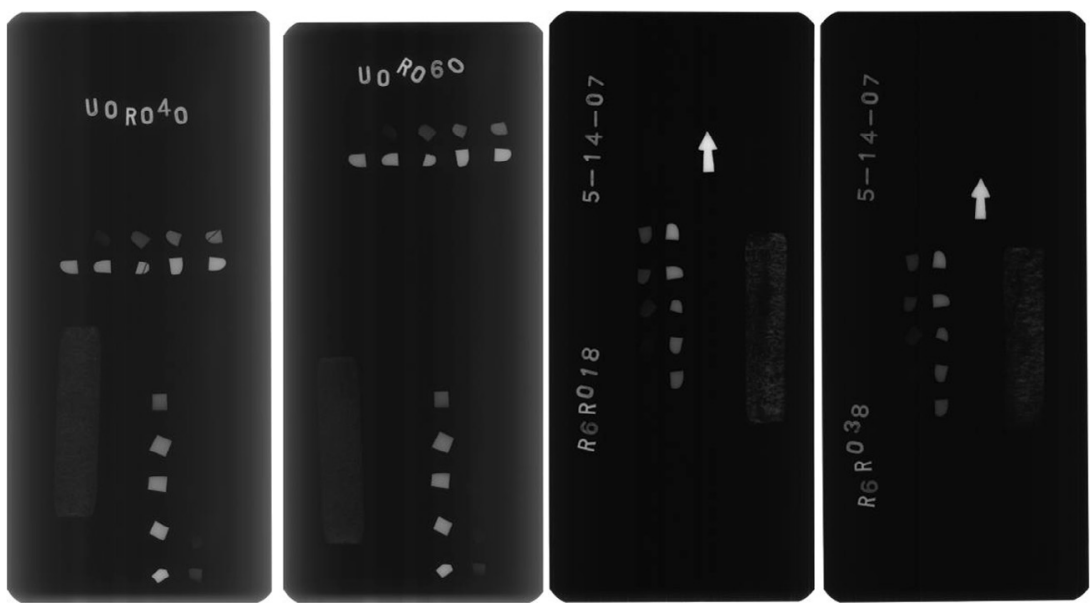

Fig. 3. Shows the four radiographs acquired for this work (plate ID of each fuel plate is tagged). 

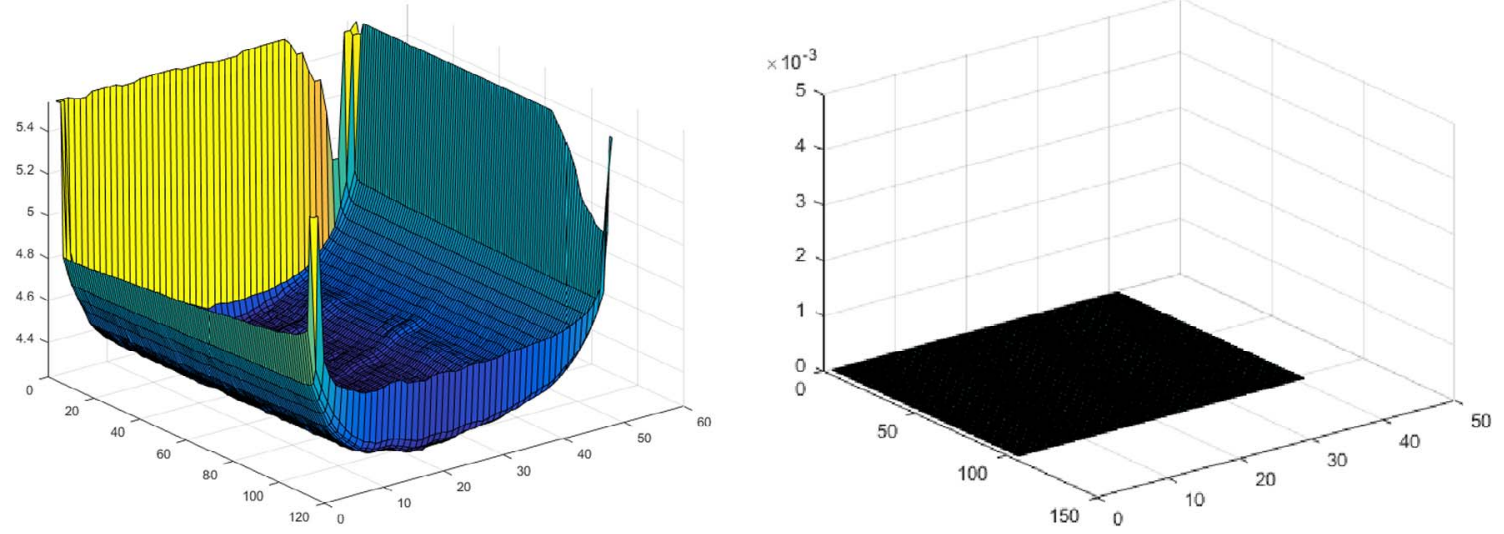

Fig. 4. Shows the estimated background (a) before and (b) after intensity inhomogeneity correction.
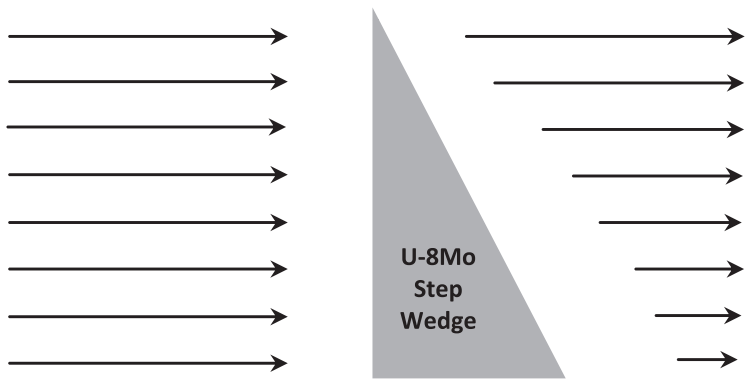

Fig. 5. Shows the X-ray attenuation in U-8Mo step wedge of varying thicknesses.

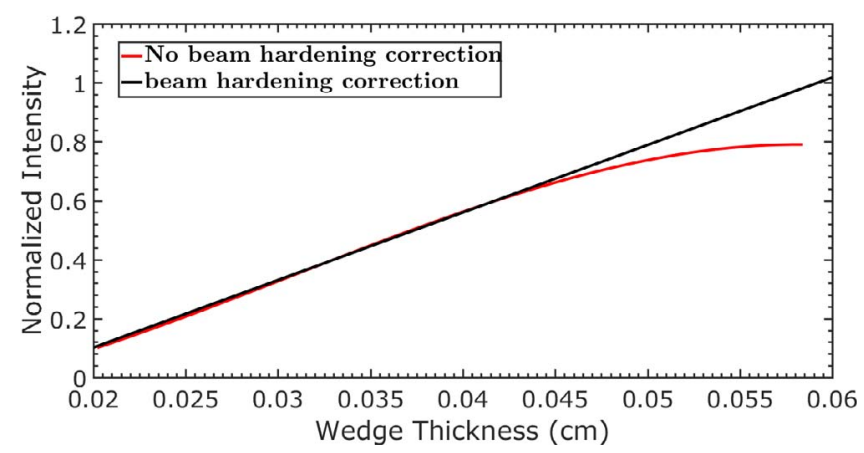

Fig. 6. Beam hardening correction using polynomial correction function.

redundant since the maximum fuel meat thickness cannot exceed the fuel plate thickness.

\subsection{Calibration}

Once the radiograph has been scanned and processed using the image processing methods described above, the absorption values are transformed into thickness values using calibration standards. The volumetric density of U-8Mo step wedge is $17.36 \mathrm{~g} / \mathrm{cm}^{3}$ while U-7Mo has $17.1 \mathrm{~g} / \mathrm{cm}^{3}$ and $\mathrm{U}_{3} \mathrm{Si}_{2}$ has $12.2 \mathrm{~g} / \mathrm{cm}^{3}$. Therefore, the effective thickness values are calculated using the following formula:

$t_{\text {foil }}=R * t_{\text {uncorrected }}$

where $R$ is the conversion factor to convert the thickness of U-8Mo to an equivalent thickness of the fuel phase in the fuel meat. $t_{\text {uncorrected }}$ is the equivalent thickness of the fuel foil using U-8Mo density standard.

$R=\frac{\rho_{\text {step wedge }}}{\rho_{\text {foil }}}$

\section{Results and discussion}

The fuel loading in any region of the mini-plate is defined by the thickness measurements taken by radiographic film measurement. Therefore, the distribution of fuel loading is calculated after the best estimation of the effective fuel meat thickness which is useful for the post-irradiation fuel swelling analysis as fuel plate growth is correlated with the local fuel loading. The inhomogeneity of the fuel loadings of $U$ $7 \mathrm{Mo}$ and $\mathrm{U}_{3} \mathrm{Si}_{2}$ miniplates are shown is Fig. 7 and Fig. 8, respectively.

Therefore, loading values are usually averaged with a $3 \mathrm{~mm} \times 3 \mathrm{~mm}$ diameter spot for quality acceptance purposes. The 3-mm spot size selected for quality inspections is dictated by the thermal hydraulic safety analysis of each reactor facility. However, fuel swelling is typically analyzed at a much higher resolution and smaller spot sizes can easily be chosen using image analysis.

The average fuel loading values using $3 \mathrm{~mm} \times 3 \mathrm{~mm}$ and $500 \mu \mathrm{m} \times 500 \mu \mathrm{m}$ diameter spots are tabulated in Table 2 . The tabulated data shows the distribution of fuel loadings of the fuel plates with
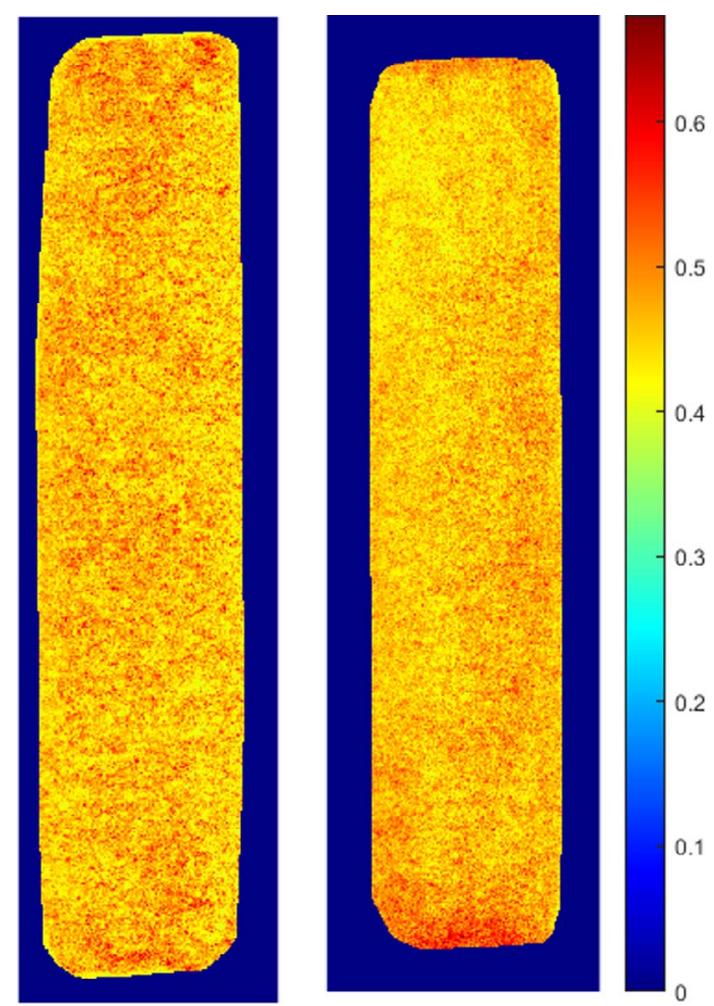

Fig. 7. Distribution of fuel loading (a) U0R040, (b) U0R060. 


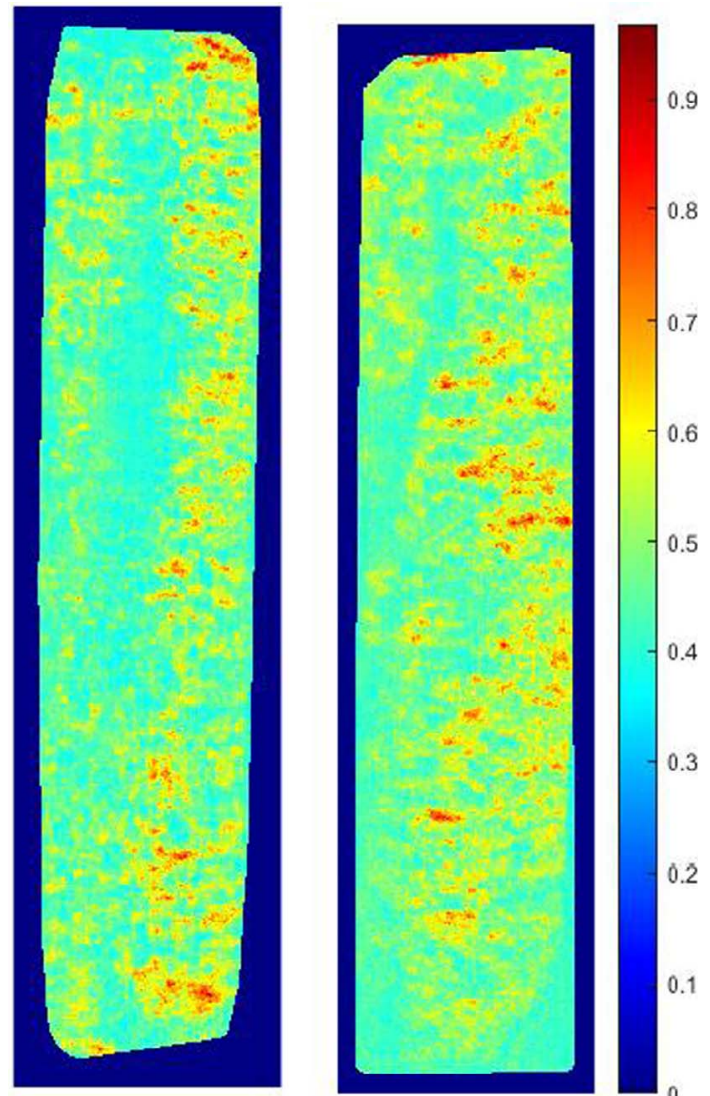

Fig. 8. Distribution of fuel loading of (a) R6R018, and (b) R6R038 plates.

Table 2

Average fuel loading values and their uncertainties.

\begin{tabular}{lll}
\hline Plate ID & $500 \mu \mathrm{m}$ Spot Size & $3 \mathrm{~mm}$ Spot Size \\
\hline U0R040 & $44.50 \% \pm 10.38 \%$ & $43.00 \% \pm 2.83 \%$ \\
U0R060 & $48.50 \% \pm 11.53 \%$ & $47.18 \% \pm 6.97 \%$ \\
R6R018 & $58.52 \% \pm 25.48 \%$ & $50.71 \% \pm 14.42 \%$ \\
R6R038 & $59.19 \% \pm 25.47 \%$ & $50.05 \% \pm 11.14 \%$ \\
\hline
\end{tabular}

an average loading and their corresponding uncertainties, assuming $100 \%$ theoretical densities. The uncertainty term gives a best estimate of how far the quantity deviates from the average value with $95 \%$ confidence. It is necessary to mention that, the determination of fuel loading using X-ray imaging is not designed for exact loading value; instead it is used to determine the variation in fuel loading.

Fig. 9 and Fig. 10 compare the distribution of fuel loadings of

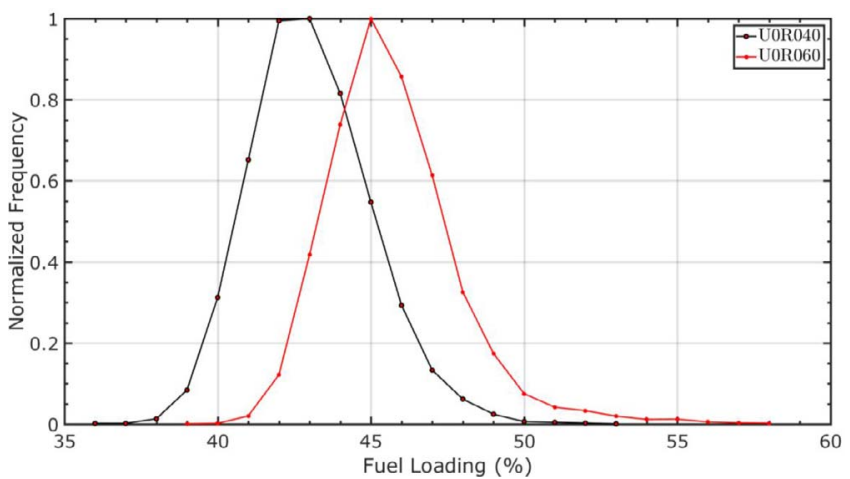

Fig. 9. Shows the distribution of uranium loading using a 500- $\mu \mathrm{m}$ spot for UOR040 and U0R060 plates.

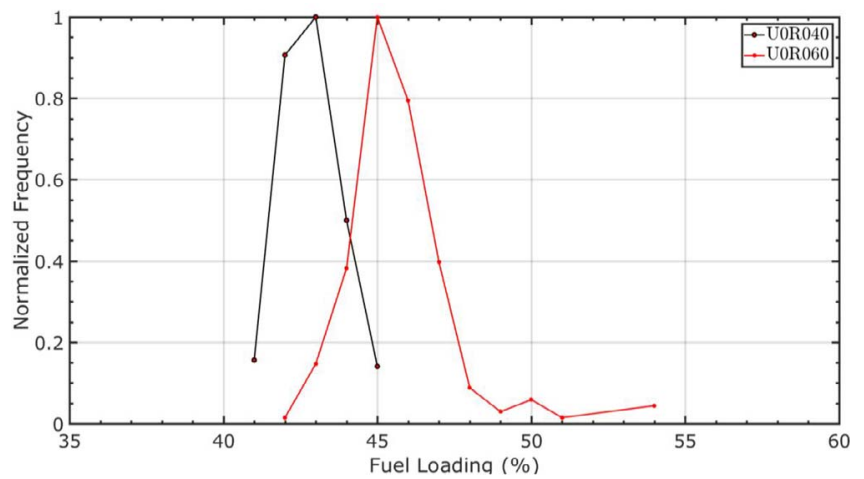

Fig. 10. Shows the distribution of uranium loading using a 3-mm spot for UOR040 and U0R060 plates.

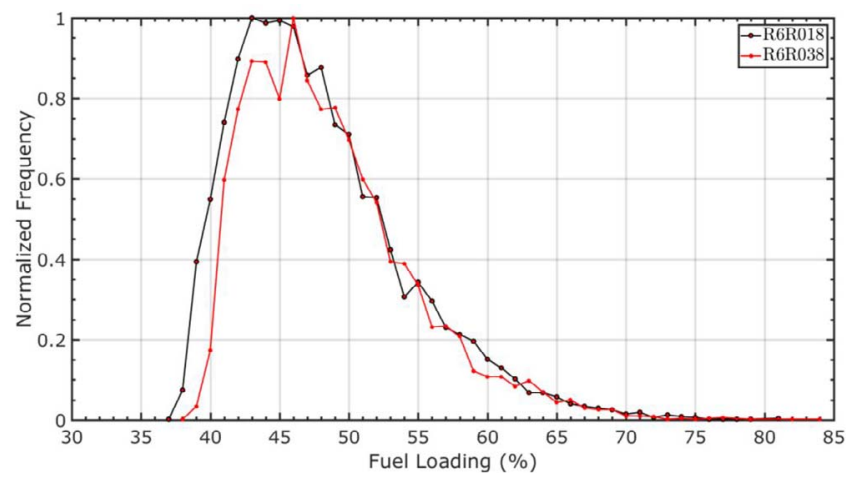

Fig. 11. Shows the distribution of uranium loading using a 500- $\mu \mathrm{m}$ spot for R6R018 and R6R038.

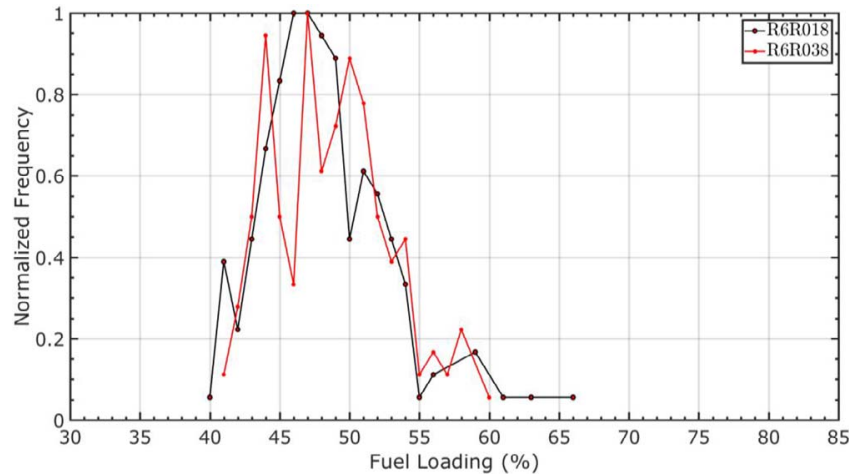

Fig. 12. Shows the distribution of uranium loading using a $3 \mathrm{~mm}$ spot for R6R018 and R6R038.

UOR040 and UOR060 plates for $500-\mu \mathrm{m}$ spot and $3 \mathrm{~mm}$ spot, respectively. The normalized peak values in the figures show the maximum occurrence of the fuel loading which is slightly different than the mean value of the loading. Similarly, Fig. 11 and Fig. 12 show the distribution of loadings of R6R018 and R6R038 plates for $500-\mu \mathrm{m}$ spot and $3 \mathrm{~mm}$ spot, respectively.

Several assumptions have been made to calculate the thickness of the miniplates. The most apparent assumption is that the composition of uranium and silicone of $\mathrm{U}_{3} \mathrm{Si}_{2}$ is assumed to be homogeneous and without impurities. This means that the attenuation coefficients of $\mathrm{U}$ and $\mathrm{Si}$ are not a function of position through the material. Second, the contribution of scattering radiation on the image is negligible therefore the build-up factor is assumed to be 1 . For photons of energy less than $1 \mathrm{MeV}$, photon interactions consist entirely of photoelectric absorptions, incoherent scatterings, and coherent scatterings. This assumption holds well for radiation that passes through the object without 
interaction, is absorbed via photoelectric absorption, or is scattered at an angle away from the film. Third, the resolution of the radiograph depends on the focal spot size and magnification. The X-ray source is assumed to be a point source. The experiment conducted in this paper utilized an X-ray point source of $4 \mathrm{~mm}$ focal spot size in diameter. The finite size of a focal spot dictates the measurable feature size in an X-ray radiograph. As the distance between the source and detector is increased, the negative effects of a large focal spot size are reduced. The ratio of source to sample distance (SSD) and sample to detector distance (SDD) is 47:1 which gives a magnification of 1.0213 . The distance between test specimen and image detector can be increased to obtain magnification in the image. However, geometric unsharpness or penumbra appears if the specimen is away from the detector.

\section{Conclusion}

Radiographic inspection provides qualitative information such as, structural integrity, as well as quantitative data such as effective fuel phase thickness and areal density. The purpose of this non-destructive study is to gain insight about qualitative and quantitative information that can be extracted from the X-ray radiographs and used as baseline characterization data in the interpretation of in-pile behavior of advanced fuel designs. Statistical analysis provides high level of confidence that the designed parameters meet the limiting criteria during fabrication. Quantitative characterization of fabricated nuclear fuels in a non-destructive approach is an effective means to support quality control techniques for nuclear fuels but can be meaningfully used to support quantitative analysis of experimental fuel plates as well. The proposed image analysis technique provides repeatability and accuracy for batch processing of fabricated samples.

\section{References}

Abir, M., Islam, F., Craft, A., Williams, W., Wachs, D., et al., 2016. Determination of optimal imaging parameters for the reconstruction of a nuclear fuel assembly using limited angle neutron tomography. J. Instrum. 11, 1-16.

Abir, M. Islam, F., Wachs, D., Lee, H., 2016. Sparse-view neutron CT reconstruction of irradiated fuel assembly using total variation minimization with Poisson statistics. J. Radioanal. Nucl. Ch. 307, 1967-1979.

Meyer, M.K., Gan, J., Jue, J.F., Keiser, D.D., Perez, E., Robinson, A., et al., 2014. Irradiation performance of U-Mo monolithic fuel. Nucl. Eng. Technol. 46, 169-182.

Tomaževič, D., Likar, B., Pernuš, F., 2002. Comparative evaluation of retrospective shading correction methods. J. Microsc. 208, 212-223.

Oberholzer, M., Östreicher, M., Christen, H., Brühlmann, M., 1996. Methods in quantitative image analysis. Histochem. Cell Biol. 105, 333-355.

Reyes-Aldasoro, C.C., 2009. Retrospective shading correction algorithm based on signal envelope estimation. Electron. Lett. 45, 454.

Beckers, A.L.D., Gelsema, E.S., De Bruijn, W.C., 1993. An efficient method for calculating the least-squares background fit in electron energy-loss spectroscopy. J. Microsc. 171, 87-92.

Likar, Maintz, Viergever, Pernuš, 2000, Retrospective shading correction based on entropy minimization. J. Microsc. 197, 285-295.

Russ, J.C., 2004. Image Analysis of Food Microstructure. CRC Press Inc. 